\title{
Provably Secure Steganography with Imperfect Sampling
}

\author{
Anna Lysyanskaya and Mira Meyerovich \\ Brown University, \\ Providence RI 02912, USA \\ \{anna, mira\}@cs.brown.edu
}

\begin{abstract}
The goal of steganography is to pass secret messages by disguising them as innocent-looking covertexts. Real world stegosystems are often broken because they make invalid assumptions about the system's ability to sample covertexts. We examine whether it is possible to weaken this assumption. By modeling the covertext distribution as a stateful Markov process, we create a sliding scale between real world and provably secure stegosystems. We also show that insufficient knowledge of past states can have catastrophic results.
\end{abstract}

Keywords: Information hiding, steganography, digital signatures, Markov processes.

\section{Introduction}

The goal of steganography is to pass secret messages by sending innocuous data. The sender may give the receiver covertexts that are distributed according to a covertext distribution. A covertext is made up of multiple documents. For example, a digital camera can define a covertext distribution of photographs, in which pixels, tiles, or even entire pictures can be considered documents. A stegosystem transforms a secret message, called a hiddentext, into a stegotext that looks like a covertext.

Real-world stegosystems are broken because they make invalid assumptions about the covertext distribution. Often, this is an assumption about an adversary's lack of knowledge about the distribution. For example, for a long time, modifying the least significant bits of pixels values in bitmaps was considered a good idea because these bits looked random. Then Moskowitz, Longdon and Chang MLC01 showed that there is a strong correlation between the least significant bit and the most significant bit (see Figures 7-10 in their paper for an instructive example).

Provably secure steganography attacks the problem by quantifying the stegosystem's need for knowledge. Anderson and Petitcolas AP98, observe that every covertext can be compressed to generate a hiddentext. Therefore, to hide a message, we can "decompress" it into a stegotext. Le [Le03] and Le and Kurosawa LK03 construct a provably secure compression-based stegosystem that assumes both the sender and receiver know the covertext distribution exactly. 
Independently, Sallee [Sal03] implemented a compression-based stegosystem for JPEG images that lets the sender and receiver estimate the covertext distribution. Compression-based schemes need to know the exact probability of every possible covertext.

Cachin Cac98 proposed using rejection-sampling to generate stegotexts that look like covertexts. A publicly known hash function assigns a bit value to documents. To send one bit, the stegosystem samples from the covertext distribution until it selects a document that evaluates to the message XOR $K$, where $K$ is a session key both parties derive from their shared secret key. Sending multiple bits requires stringing several documents together. Cachin's scheme is secure if the hash function is unbiased. Because the stegosystem only needs to be able to sample from the covertext distribution, it is known as a black-box stegosystem. This paper examines the nature of the black-box required for steganography.

Hopper, Langford and von Ahn HLvA02 improve on Cachin's results. They give the first rigorous definition of steganographic security by putting it in terms of computational indistinguishability from the covertext distribution. Their stegosystem uses Cachin's rejection-sampling technique, but generalizes it to be applicable to any distribution, assuming it (1) has sufficient entropy and (2) can be sampled perfectly based on prior history. Reyzin and Russell [RR03 improve the robustness and efficiency of the Hopper et al. scheme. Von Ahn and Hopper vAH04 create a public-key provably secure stegosystem and Backes and Cachin [BC05 and Hopper Hop05 consider chosen covertext attacks. Despite these improvements, the two assumptions necessary for provably secure steganography remain in the literature. The entropy assumption appears inherent to the problem. We address the possibility of weakening the sampling assumption.

Some prior work focuses on the performance measures of black-box stegosystems. In particular, there is the rate of a stegosystem, which measures how many bits of the message you can pack per document transmitted. There is also the query complexity per document which measures how many times you need to query the sampler in order to create a document of the stegotext. Notably, Dedic et al. DIRR05] showed that if the rate is $w$, then the query complexity per document is $2^{w}$. We do not worry about query complexity, but rather about the very nature of the sampler at the disposal of a stegosystem, so the underlying question is very different.

Black-box stegosystems [Cac98, HLvA02, RR03, vAH04, BC05, Hop05] assume that they have access to an adaptive sampler. The sampler must be able to take an arbitrary history of documents as input and output a document distributed according to the covertext distribution conditioned on the prior history. For example, if our covertext distribution consists of images of teddy-bears, and each document is an $8 \times 8$ pixel tile, then the sampler's input is the first $k-1$ tiles of the image (say, the ears of the teddy bear), and the output is the $k^{\text {th }}$ tile of the image (say, the nose). The stegosystem needs to be able to query the sampler multiple times on the same input: it continues to sample until it gets a document that corresponds to the message it wants to hide. The sampler must output many noses that correspond to the same set of ears. 
Sampling teddy-bear noses based on teddy-bear ears is an absurd example. We use it because in the real world there are no known naturally occuring distributions that can be sampled based on history 1 Our work examines whether accurate adaptive sampling is really neccessary. We come to the somewhat unsurprising conclusion that a stegosystem must assume that the sampler it uses is accurate. Our chief contribution is to examine what it really means to have a bad sampler.

There are many ways to characterize the abilities of a sampler. It can be contextual: given documents $d_{i}, \ldots, d_{j-1}, d_{j+1}, \ldots, d_{k}$, it produces possible values for $d_{j}$. A special case of a context sampler is a history-based sampler: given $d_{i}, \ldots, d_{j-1}$, it produces possible values for $d_{j}$. Since history-based samplers are sufficient for secure steganography, we limit our examination to those. Past experience has shown that stegosystems are broken when there is a statistical correlation between documents of the covertext distribution. For example, the least-significant and most-significant bits in a bitmap are correlated, which leads to Moskowitz et al's MLC01 attack. Therefore, a history-based sampler might make a mistake when it does not consider some of the history (usually, due to either ignorance or memory and computational limitations). This means we can characterize a history-based sampler by the length of history it considers. We call a sampler that considers only some of the history a semi-adaptive sampler, while one that ignores the history entirely is called non-adaptive.

Some samplers may be limited by the number of times they can be queried on the same input. For example, Hopper et al HLvA02 point out that human beings have difficulty generating multiple independent samples of e-mails on the same topic. The distribution of the output of the sampler and the covertext distribution may gradually (or even sharply) diverge after several draws. This problem can be analyzed in terms of query complexity, which is discussed in DIRR05. We do not consider it further.

Semi-adaptive samplers lead us naturally to consider Markov processes. Suppose the actual covertext distribution is $D$. The distribution $D^{\prime}$ from which a semi-adaptive sampler draws is a Markov process. Since a stegosystem approximates the distribution it samples, security requires that $D$ and $D^{\prime}$ are sufficiently close. We introduce the concept of an $\alpha$-memoryless distribution, a distribution that is computationally indistinguishable from some Markov process of order $\alpha$. We design the definition of $\alpha$-memorylessness so that it is necessary and sufficient for secure black-box steganography with semi-adaptive sampling.

We have three results:

1. We analyze what happens to the von Ahn and Hopper public key stegosystem vAH04 when the sampler only considers the last $\alpha$ documents of the history. We calculate how inaccuracy in the sampler translates into insecurity in the stegosystem. Our results show that assuming the covertext distribution is $\alpha$-memoryless is neccessary and sufficient for maintaining security.

\footnotetext{
${ }^{1}$ Artificial distributions, such as the output of randomized algorithms and encryption functions, can be sampled perfectly. However, they tend to arouse suspicion, thus making them unsuitable for steganography.
} 
2. We analyze the security of non-adaptive black-box stegosystems. Independently 2 Petrowski et al. PKSM] implemented a non-adaptive stegosystem for JPEG images, giving empirical evidence that memoryless distributions exist and can be used for secure steganography.

3. We construct a pathological $\alpha$-memoryless high-entropy distribution for which black-box steganography is infeasible if the stegosystem's sampler considers only the last $\alpha-1$ documents of the history (under the discrete logarithm assumption). An efficient adversary can detect any attempt at covert communication with overwhelming probability.

Organization: Section 2 presents notation and definitions. Section 3 analyzes the von Ahn and Hopper stegosystem vAH04 in the context of semi-adaptive sampling. Section 4 examines non-adaptive stegosystems. Section 5 constructs a pathological covertext distribution for which black-box steganography is infeasible. Section 6 concludes. We have omitted some of the proofs; they can be found in the full paper [LM05].

\section{Notation}

We call a function $\nu: \mathbb{N} \rightarrow(0,1)$ negligible if for all $c>0$ and for all sufficiently large $k, \nu(k)<1 / k^{c}$.

The hiddentext will always be in $\{0,1\}^{*}$. A covertext is composed of a sequence of documents. Each document comes from the alphabet $\mathbb{A} ;|\mathbb{A}|$ may be exponential. We denote concatenation with the o operator; a string $s$ can be parsed to $s=s_{1} \circ s_{2} \circ \ldots \circ s_{n}$, where $|s|=n$. The symbol $\lambda$ denotes the empty string.

Our main results measure the security of stegosystems; we calculate the probability of a stegosystem being broken in terms of the probability of an adversary breaking other cryptographic primitives. The term $\mathbf{A d v}_{P}^{\text {game }}(A, k)$ refers to the probability of adversary $A$ breaking the security of primitive $P$ in the context of a scenario defined by game when the security parameter is $k$. For example, $\mathbf{A d v}_{\text {DSA }}^{\text {sig }}(A, 160)$ is the probability that $A$ forges a 160 -bit DSA signature. What we really care about is attacks by an a large class of adversaries, where each class defines the maximum amount of time and other resources an adversary can use. InSec ${ }_{P}^{\text {game }}$ (class) is the maximum probability that any adversary in class can break the security of primitive $P$ while in the scenario defined by game. For example, $\operatorname{InSec}_{F}^{\text {owf }}(t, k)$ is the maximum probability of any adversary inverting the one-way function $F$ if it runs in $t(k)$ time, where $k$ is the security parameter. Therefore, if we say $3 \operatorname{InSec} \operatorname{Sec}_{\Sigma}^{\text {sig }}(t, q, k) \leq \operatorname{InSec}_{F}^{\text {owf }}(t, k)$, this means that signature scheme $\Sigma$ is three times as hard to break as one-way function $F$.

To define the probability of an attacker winning in a scenario, we need to consider the outcome of several events. The expression $P f\left[e_{1}, e_{2}, \ldots, e_{n}: c\right]$ is the probability that condition $c$ holds given that events $e_{1}, e_{2}, \ldots, e_{n}$ occured (and in that order). For example, let $A$ be some algorithm that takes as input an integer

${ }^{2}$ We presented preliminary results of this work in August 2004 [LM04]. 
and outputs a single bit. The expression $\operatorname{Pr}[x \leftarrow \mathbb{Z} ; b \leftarrow A(x): b=x \bmod 2]$ is the probability that $b=x \bmod 2$, given that first $x$ was randomly chosen from $\mathbb{Z}$ and then $b$ was generated by executing $A(x)$. In other words, it is the probability that $A$ correctly calculates $x \bmod 2$ on a randomly chosen integer $x$.

We say that a function $f: \mathbb{A} \rightarrow\{0,1\}$ is $\epsilon$-biased with respect to distribution $D$ if $|\operatorname{Pr}[d \leftarrow D: f(d)=0]-1 / 2|<\epsilon$. A $\epsilon(k)$-biased function is called an unbiased function if $\epsilon$ is a negligible function 3 A covertext distribution that has sufficient minimum entropy for steganography is called always informative (see Hopper et al HLvA02] for details).

We write $x \leftarrow D\langle h, n\rangle$ to denote sampling $n$ documents from $D$ conditioned on the prior history $h ; D\langle h, n\rangle$ defines a distribution over $\mathbb{A}^{n}$. A semi-adaptive sampler samples one document from the distribution $D$ conditioned only on the last $\alpha$ documents of $h . D^{\alpha}\langle h, n\rangle$ generates an $n$-document string by calling a semi-adaptive samper $n$ times, each time appending the result to $h$. When we give a player sampling access to a distribution, we use $\cdot$ to denote the parameters that the player can pick. For example, the oracle $D\langle\cdot, 2\rangle$ samples two documents from $D$ based on a history supplied by the player.

An $\alpha$-memoryless distribution is indistinguishable from a Markov process of order $\alpha$. (A sequence of random variables $X_{1}, \ldots, X_{n}$ such that for $\alpha<i \leq n$, the conditional distribution $\left\{X_{i} \mid X_{i-\alpha}, \ldots, X_{i-1}\right\}$ is identical to the conditional distribution $\left\{X_{i} \mid X_{1}, \ldots, X_{i-1}\right\}$.) Since we require computational indistinguishability, we parameterize everything by $k$ (e.g. $D_{k}$, a family of distributions).

Definition 1 ( $\alpha$-Memoryless). Let $D_{k}$ be a family of distributions indexed by a public parameter $k$ and let $D_{k}^{\alpha}$ be the best Markov model of order $\alpha$ that approximates $D_{k}$. We define the advantage of an adversary $A$ against the Markov model as:

$$
\begin{aligned}
\operatorname{Adv}_{D, \alpha}^{\mathrm{mem}}(A, k)=\mid \operatorname{Pr}\left[h \leftarrow D_{k}\langle\lambda, n(k)-1\rangle\right. & \left.; x \leftarrow D_{k}^{\alpha}\langle h, 1\rangle: A(h \circ x)=1\right] \\
& -\operatorname{Pr}\left[x \leftarrow D_{k}\langle\lambda, n(k)\rangle: A(x)=1\right] \mid
\end{aligned}
$$

We let $\operatorname{InSec}_{D, \alpha}^{\text {mem }}(t, n, k)=\max _{A \in \mathcal{A}(t, n, k)} \mathbf{A d v}_{D, \alpha}^{\text {mem }}(A, k)$, where $\mathcal{A}(t, n, k)$ is the set of all adversaries that run in time $t(k)$ and get a sample $n(k)$ documents long. We say that $D_{k}$ is $\alpha$-memoryless if $\operatorname{InSec}{ }_{D, \alpha}^{\text {mem }}(t, n, k) \leq \nu(k)$ for some negligible function $\nu . D_{k}$ is strictly $\alpha$-memoryless if $\operatorname{InSec}_{D, \beta}^{\operatorname{mem}}(t, n, k)$ is non-negligible for all $\beta<\alpha$.

Remark 1. This property is necessary and sufficient for steganography with semiadaptive sampling.

The following definitions are either standard or come from von Ahn and Hopper vAH04. We assume that all adversaries are probabilistic polynomial-time Turing machines. However, the distributions we work with are arbitrary and may act as arbitrarily powerful adversaries. For example, someone who can adaptively sample a distribution might be able to use it to calculate discrete logarithms.

${ }^{3}$ The function $f$ is typically chosen after we fix the distribution (and the security parameter). A universal hash function is often used in practice. 
We define $\operatorname{InSec}_{X, Y}^{\text {dist }}(t, n, k)$ as the maximum probability that an adversary can distinguish distribution $X_{k}$ from $Y_{k}$ if it runs in time $t(k)$ and gets a $n(k)$ document long sample. Steganography requires an IND\$-CPA cryptosystem whose ciphertext is indistinguishable from random. $\operatorname{InSec}_{\mathcal{E}}^{\text {cpa }}(t, q, n, k)$ is the insecurity of cryptosystem $\mathcal{E}$ against a chosen plaintext attack by an adversary that runs in $t(k)$ time, makes $q(k)$ queries and gets responses totaling $n(k)$ bits (see Hopper et al. HLvA02] or full paper for details).

The standard specification [vAH04] of a public-key stegosystem is:

Definition 2 (Public Key Stegosystem). A public key stegosystem is the triple $\mathcal{S}=(S G, S E, S D) . S G\left(1^{k}\right)$ generates a key-pair $(S K, P K) . S E(P K, m)$ takes the public key $P K$ and a message $m \in\{0,1\}^{*}$, and returns some stegotext s. $S D(S K, s)$ takes the secret key $S K$ and stegotext $s$ and returns a hiddentext $m$. For all $m \in\{0,1\}^{*}$, the probability that $S D(S K, S E(P K, m))$ fails to recover $m$ should be negligible.

Von Ahn and Hopper vAH04 define the security of a public-key stegosystem against a chosen hiddentext attack. An adversary $A$ queries an oracle with hiddentexts. The oracle responds either with stegotexts generated by $S E(P K, \cdot)$ or with covertexts of the appropriate length, generated by $D^{*}(\cdot)$. A should not be able to distinguish the two cases.

Definition 3 (SS-CHA). The advantage of an adversary A against a publickey stegosystem $\mathcal{S}=(S G, S E, S D)$ in a chosen hiddentext attack (CHA) is:

$$
\operatorname{Adv}_{\mathcal{S}, D}^{\text {cha }}(A, k)=\left|\operatorname{Pr}\left[\operatorname{PK} \leftarrow S G\left(1^{k}\right): A_{k}^{S E(P K, \cdot), D}=1\right]-\operatorname{Pr}\left[A_{k}^{D^{*}(\cdot), D}=1\right]\right|
$$

We let $\operatorname{InSec}_{\mathcal{S}, D}^{\text {cha }}(t, q, n, k)=\max _{A \in \mathcal{A}(t, q, n, k)} \mathbf{A d v}_{\mathcal{S}, D}^{\text {cha }}(A, k)$ where $\mathcal{A}(t, q, n, k)$ $i s$ the set of all adversaries that run in $t(k)$ time, make $q(k)$ queries and get responses totaling $n(k)$ bits. A stegosystem is considered secure against a chosen hiddentext attack (SS-CHA) if $\operatorname{InSec}_{\mathcal{S}, D}^{\text {cha }}(t, q, n, k) \leq \nu(k)$ for some negligible function $\nu$.

Remark 2. We restrict the usual definition of security. Typically, the adversary is allowed to query the stegosystem with any history and message. In our model, we assume that an adaptive sampler does not exist. A stegosystem that is secure against such an attack is an adaptive sampler (see Hopper Hop04 Section 3.3.2). We force the adversary to always query the stegosystem with history $\lambda$ (the empty string).

\section{Semi-adaptive Stegosystem}

In this section we examine what happens to the von Ahn and Hopper vAH04 public-key stegosystem when we replace the adaptive sampling oracle with a semi-adaptive one. We show that if the oracle samples based on the last $\alpha$ documents of the history, then an $\alpha$-memoryless distribution is necessary and sufficent for maintaining security. 


\subsection{The vAH04 Stegosystem with Semi-adaptive Sampling}

The von Ahn and Hopper stegosystem [vAH04] (Construction 2 in their paper) is a public-key provably secure stegosystem; See Algorithm 3,1 . and 3,2 for the encoding and decoding algorithms (we have modified them slightly to fit our notation). Their stegosystem uses an IND $\$$-CPA public-key cryptosystem $\mathcal{E}=$ $\left(G, E_{P K}, D_{S K}\right)$ and a publicly known function $f: \Sigma \rightarrow\{0,1\}$ that is $\epsilon$-biased with respect to the covertext distribution $D_{k}$. The encoder first encrypts the message using $E_{P K}$. Next, for each bit $b$ of ciphertext, the encoder samples the covertext distribution until it gets a document $d$ such that $f(d)=b$. The encoder appends all of the resulting documents together to form the stegotext. The decoder extracts the ciphertext by evaluating $f$ on every document of the stegotext and then decrypts the ciphertext.
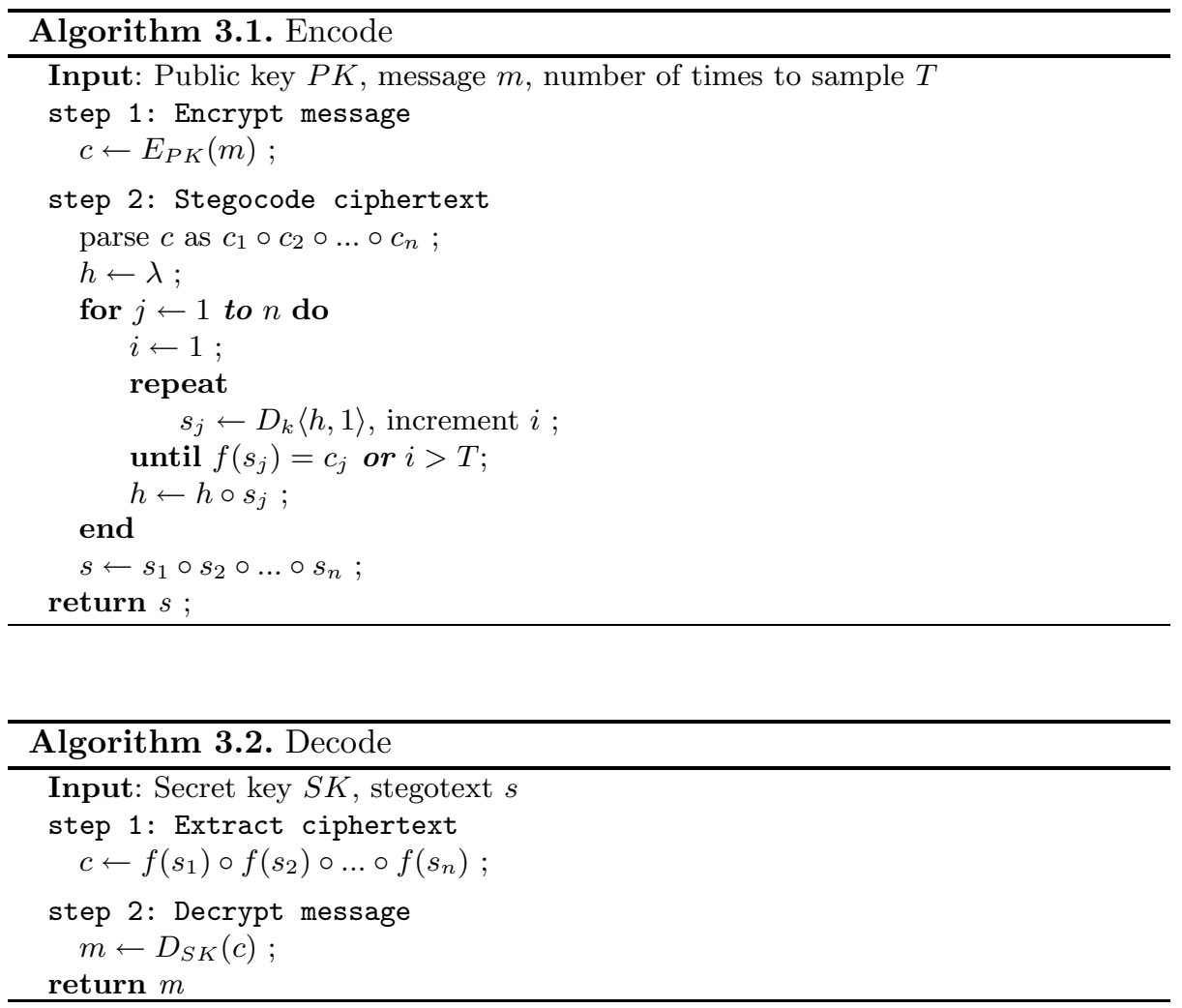

For the remainder of Section [3, we will refer to the von Ahn and Hopper stegosystem as $\mathcal{S}=(S G, S E, S D)$ and assume that $D_{k}$ is the covertext distribution. We define a length function $\mathcal{L}: \mathbb{Z} \rightarrow \mathbb{Z}$ that calculates the length of a ciphertext for a message $m: \mathcal{L}(|m|)=\left|E_{P K}(m)\right|$. Von Ahn and Hopper vAH04 prove that their stegosystem is secure: 
Theorem 1 ( $\mathbf{\text { AH04 }}$ ). If $D_{k}$ is an always informative distribution and $f$ is $\epsilon$-biased on $D_{k}$, then $\mathcal{S}$ is a $S S$-CHA secure stegosystem:

$$
\operatorname{InSec}_{\mathcal{S}, D}^{\text {cha }}(t, q, n, k) \leq \operatorname{InSec}_{\mathcal{E}}^{\text {cpa }}(t+O(k n), q, n, k)+\mathcal{L}(n) \epsilon
$$

Remark 3. What Theorem 1 really states is that the output of $\mathcal{S}$ is indistinguishable from the distribution it samples.

$\mathcal{S}$ uses a perfect sampler. We now consider the stegosystem $\mathcal{T}=(T G, T E, T D)$ that functions identically to $\mathcal{S}$, except that its only access to $D_{k}$ is via $D_{k}^{\alpha}$, an oracle that only considers the last $\alpha$ documents of the history. The main result of this section is the proof that $\mathcal{T}$ is correct and that $\mathcal{T}$ is secure if and only if $D_{k}$ is $\alpha$-memoryless.

\subsection{Analysis of $\mathcal{T}$}

Lemma 1. Assume that $D_{k}$ is an always informative $\alpha$-memoryless distribution and $f$ is an $\epsilon$-biased function on $D_{k}$. For all hiddentexts $m \in\{0,1\}^{*}$, the probability that $\mathcal{T}$ fails to encode $m$ is negligible:

$$
\begin{aligned}
\operatorname{Pr}\left[(P K, S K) \leftarrow T G\left(1^{k}\right) ; s\right. & \left.\leftarrow T E(P K, m) ; m^{\prime} \leftarrow T D(S K, s): m^{\prime} \neq m\right] \\
& \leq \mathcal{L}(|m|)\left(1 / 2+\epsilon+\operatorname{InSec}_{D, \alpha}^{\text {mem }}(O(1), \mathcal{L}(|m|), k)\right)^{k}
\end{aligned}
$$

Proof. The probability of error is at most the length of the ciphertext multiplied by the probability that any individual bit of ciphertext is encoded incorrectly. See full paper for details.

Theorem 2. If $D_{k}$ is an always informative $\alpha$-memoryless distribution and $f$ is $\epsilon$-biased on $D_{k}$, then $\mathcal{T}$ is a $S S$-CHA secure stegosystem:

$$
\begin{aligned}
\operatorname{InSec}_{\mathcal{T}, D}^{\text {cha }}(t, q, n, k) \leq \operatorname{InSec}_{\mathcal{E}}^{\text {cpa }}(t+ & O(k n), q, n, k) \\
& +n \operatorname{InSec}_{D, \alpha}^{\text {mem }}(t+O(n), n, k)+\mathcal{L}(n) \epsilon
\end{aligned}
$$

Proof. The probability that $\mathcal{T}$ can be broken is the probability that an adversary distinguishes the IND $\$$-CPA cryptosystem $\mathcal{E}$ from random plus the probability that an adversary can distinguish $D_{k}$ from $D_{k}^{\alpha}$; both these values are negligible. See full paper for details.

Theorem 3. Let $D_{k}$ be an always informative distribution and $f$ an $\epsilon$-biased function on $D_{k}$. If $D_{k}$ is not $\alpha$-memoryless then $\mathcal{T}$ is not a SS-CHA secure stegosystem:

$$
\begin{aligned}
\operatorname{InSec}_{\mathcal{T}, D}^{\text {cha }}(t+O(1), 1, n, k) \geq \operatorname{InSec}_{D, \alpha}^{\text {mem }}(t, n, k) & \\
& -\operatorname{InSec}_{\mathcal{E}}^{\text {cpa }}(t+O(k n), 1, n, k)-n \epsilon
\end{aligned}
$$

${ }^{4}$ As a mnemonic device, think of $\mathcal{S}$ as the stegosystem with a Standard sampler and $\mathcal{T}$ as having a sampler that considers only the Tail of the history. 
Remark 4. Note that $\operatorname{InSec}_{D, \alpha}^{\text {mem }}(t, n, k)$ is not negligible because $D_{k}$ is not $\alpha$ memoryless. Any adversary that can distinguish $D_{k}$ from $D_{k}^{\alpha}$ can be used to attack $\mathcal{T}$.

Proof. Assume $D_{k}$ is not $\alpha$-memoryless. By definition, there exists an adversary $A$ such that $\mathbf{A d v}_{D, \alpha}^{\text {mem }}(A, k)$ is non-negligible. Let $A$ run in time $t$ and require a challenge sample of length $n$. We use $A$ to create an adversary $B$ that can tell whether it is querying an oracle representing $\mathcal{T}$ or $D_{k} . B$ will ask its oracle for a single covertext of length $n$ and pass the output to $A$. $B$ will output whatever $A$ outputs. $B$ 's advantage in distinguishing $\mathcal{T}$ from $D_{k}$ is at least as much as $A$ 's advantage in distinguishing $D_{k}^{\alpha}$ from $D_{k}$ minus the probability of distinguishing $\mathcal{T}$ from $D_{k}^{\alpha}$ :

$$
\mathbf{A d v}_{\mathcal{T}, D}^{\text {cha }}(B, k) \geq \mathbf{A d v}_{D, \alpha}^{\text {mem }}(A, k)-\mathbf{I n S e c}_{\mathcal{T}, D^{\alpha}}^{\text {cha }}(t, 1, n, k)
$$

Using Theorem 1, we get:

$$
\mathbf{A d v}_{\mathcal{T}, D}^{\text {cha }}(B, k) \geq \mathbf{A d v}_{D, \alpha}^{\text {mem }}(A, k)-\operatorname{InSec}_{\mathcal{E}}^{\text {cpa }}(t+O(k n), 1, n, k)-n \epsilon
$$

$B$ runs in time $t+O(1)$ and gets 1 challenge string of length $n$, therefore:

$$
\begin{aligned}
\operatorname{InSec}_{S, D}^{\mathrm{cha}}(t+O(1), 1, n, k) \geq \operatorname{InSec}_{D, \alpha}^{\mathrm{mem}}( & t, n, k) \\
& -\operatorname{InSec}_{\mathcal{E}}^{\mathrm{cpa}}(t+O(k n), 1, n, k)-n \epsilon
\end{aligned}
$$

This means that if $D_{k}$ is not $\alpha$-memoryless, then there exists an adversary that can launch a successful SS-CHA attack on $\mathcal{T}$ with non-negligible probability.

Remark 5. The above proof would probably work for any black-box stegosystem. However, because it is unclear how to deal with a stegosystem that somehow uses outside information (or how to rule out this possibility), we limit our analysis to the stegosystem $\mathcal{T}$.

\section{Non-adaptive Stegosystems}

In this section, we show how to apply public-key black-box steganography as proposed by von Ahn and Hopper vAH04 to real world covertext distributions. (Independently, Petrowski et. al. PKSM implemented a similar system for JPEG images, but their work has no security analysis.) The key insight is that multiple digital photographs of a still scene are almost but not completely identical. We can break up each image into $8 \times 8$ pixel tiles 5 A cryptographic hash function assigns a value to each tile. The stegosystem choses the appropriate tiles to create a composite photo that encodes the secret message. The scheme assumes each $8 \times 8$ pixel tile is independent of its neighbors.

This stegosystem is equivalent to using $D_{k}^{0}$ to sample $D_{k}$ and assuming that the covertext distribution is 0-memoryless, as shown in Algorithm 4.1. Nonadaptive steganography can be applied to any digital image format, TCP timestamp intervals, etc.

\footnotetext{
${ }^{5}$ The dimensions of the tile are an artifact of the JPEG compression algorithm.
} 


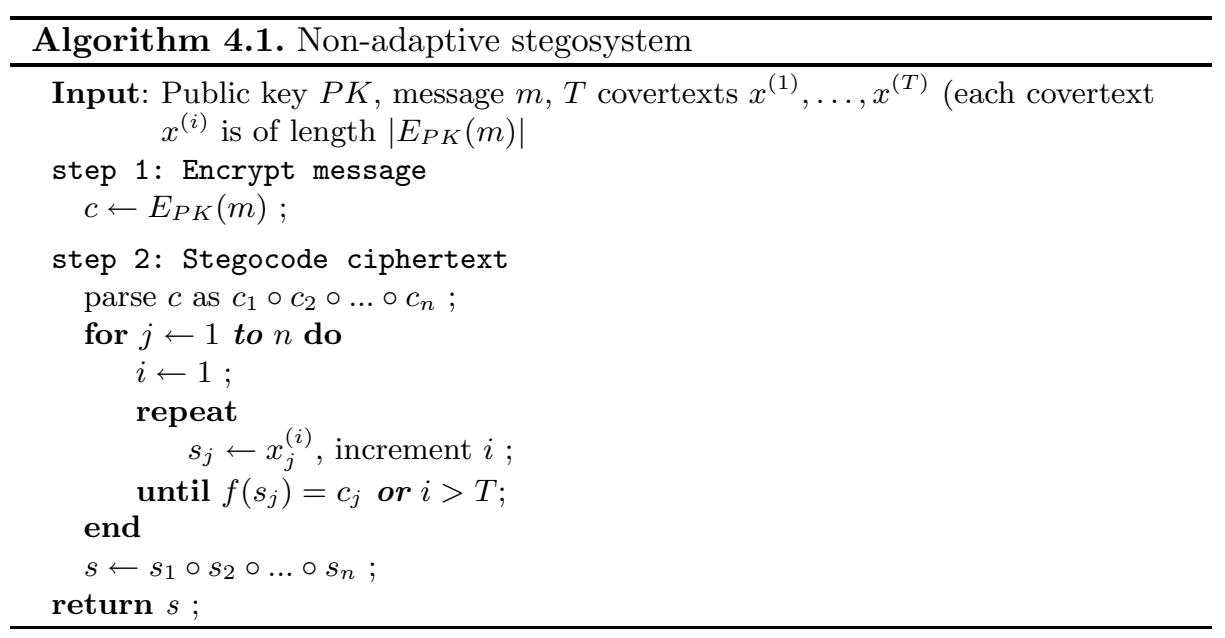

The analysis of Algorithm 4.1 follows directly from Section 3 , Correctness: The probability that the stegosystem fails to encode a hiddentext $m$ is: $\mathcal{L}(|m|)(1 / 2+$ $\left.\epsilon+\operatorname{InSec}_{D, 0}^{\text {mem }}(O(1), \mathcal{L}(|m|), k)\right)^{k}$. Security: Algorithm 4.1 is secure if and only if $D$ is 0-memoryless: an independent, but not necessarily identically distributed, sequence of random variables.

\section{Pathological Covertext Distribution}

In this section, we construct a pathological strictly $\alpha$-memoryless distribution and prove that no computationally bounded algorithm can use it to hide messages without access to $D_{k}^{\alpha}$. The distribution will publish a verification key that can be used by anyone to check if a covertext is legitimate. The probability that steganography will be detected is $1-\nu(k)$, where $\nu$ is a negligible function.

We give a stegosystem a list of covertexts generated by $D\langle\lambda, \cdot\rangle$ and access to $D^{\alpha-1}\langle\cdot, 1\rangle$, a semi-adaptive oracle with insufficient memory. For example, a stegosystem might store a database of photographs (this corresponds to $D\langle\lambda, \cdot\rangle$ ) and maintain an internal Markov model about pixel color distributions based on the 8 adjacent pixels (this corresponds to $D^{\alpha-1}\langle\cdot, 1\rangle$, where $\alpha-1=8$ ). We show that any stegotext produced by a stegosystem is really just a quote of a covertext in its database.

\subsection{The Distribution}

Our goal is to devise a covertext distribution where (1) each document depends on only the $\alpha$ documents that came before it (so it is $\alpha$-memoryless); (2) a stegosystem cannot by itself compute the $i$ th document $d_{i}$ in a legitimate covertext; finally (3) it is very unlikely that the output of $D^{\alpha-1}\langle h, 1\rangle$ is a valid continuation of the last $\alpha$ documents of $h$. 
The first construction that comes to mind is to make each document be a concatenation of a random number $r_{i}$ and a signature on the previous $\alpha$ random numbers: $\sigma_{i}=\sigma\left(r_{i-\alpha}, \ldots, r_{i}\right)$. This will meet requirements (1) and (2). There is a subtle problem with this as far as requirement (3) is concerned. Suppose we are given $\alpha-1$ documents $r_{n-\alpha+1} \sigma_{n-\alpha+1}, \ldots, r_{n-1} \sigma_{n-1}$. The signatures $\sigma_{n-\alpha+1}, \ldots, \sigma_{n-1}$ can leak partial information about the value $r_{n-\alpha}$. As a result, $D^{\alpha-1}\langle\cdot, 1\rangle$, even though not explicitly given $d_{n-\alpha}$, may nevertheless calculate $r_{n-\alpha}$ and compute the correct signature $\sigma_{n}=\sigma\left(r_{n-\alpha}, \ldots, r_{n}\right)$.

In order to fix this problem, we need to construct a signature function $\sigma$ for which the following property holds: We fix a sequence of $2 \alpha-1$ integers $r_{1}, \ldots, r_{2 \alpha-1}$. Then the sequence of $\alpha-1$ documents $r_{\alpha+1} \sigma_{\alpha+1}, \ldots, r_{2 \alpha-1} \sigma_{2 \alpha-1}$ should be information theoretically independent of $r_{\alpha}$. This property ensures that $D^{\alpha-1}$ cannot learn $r_{\alpha}$ and so will be unable to compute the correct signature $\sigma_{2 \alpha}$ based on the previous $\alpha$ documents of $h$, as required by (3) above.

Consider the following hash function $h: \mathbb{Z}_{p}^{\alpha} \rightarrow G$, where $p$ is a $k$-bit prime

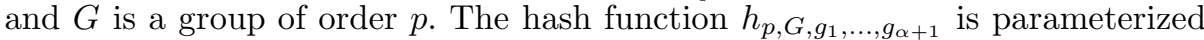
by $p, G$ and $\alpha+1$ generators of $G: g_{1}, \ldots, g_{\alpha+1}$. (We will omit the subscript of $h$ in the future). On input $\left(r_{1}, \ldots, r_{\alpha+1}\right) \in \mathbb{Z}_{p}^{\alpha+1}$ the hash function returns:

$$
h\left(r_{1}, r_{2}, \ldots, r_{\alpha+1}\right) \doteq g_{1}^{r_{1}} \cdot g_{2}^{r_{2}} \cdots \cdot g_{\alpha+1}^{r_{\alpha+1}}
$$

The hash function $h$ has the information hiding property that we need because it reveals only a linear combination of its inputs (see the proof of Lemma 4 in the full paper).

We now formalize the above discussion. We define a secure stateless signature scheme, show how to combine it with $h$ and prove the result is secure under the discrete logarithm assumption. Then we construct our pathological distribution.

Definition 4 (Stateless Signature Scheme). A stateless signature scheme $\Sigma=(G, \sigma, V)$ is a triple of polynomial time algorithms where: $G\left(1^{k}\right)$ is the key generation algorithm, $\sigma:\{0,1\}^{k} \times \mathcal{M}_{k} \rightarrow\{0,1\}^{\text {poly }(k)}$ is a probabilitic algorithm that on input $(S K, m)$ outputs a poly $(k)$ bit signature, and $V:\{0,1\}^{k} \times \mathcal{M}_{k} \times$ $\{0,1\}^{\text {poly }(k)} \rightarrow\{0,1\}$ is the signature verification function that accepts valid signatures.

We define $\operatorname{InSec}_{\Sigma}^{\text {sig }}(t, q, k)$ as the insecurity of signature scheme $\Sigma$ against an adaptive chosen message attack by an adversary that runs in time $t(k)$ and makes $q(k)$ queries to the signing oracle (see Goldreich Gol04 for details).

Goldreich Gol04 shows that stateless signature schemes exist if one-way functions exist. It is also known that the discrete logarithm assumption implies oneway functions. Therefore, the discrete logarithm assumption also implies the existence of stateless signature schemes. We let $\mathbf{D L}(t, k)$ be the maximum probability that any algorithm running in time $t(k)$ can solve the discrete logarithm problem. 
We construct a signature scheme using the hash function $h$ :

Construction 1. Let $\Sigma^{\prime}=\left(G^{\prime}, \sigma^{\prime}, V^{\prime}\right)$ be a secure stateless signature scheme that takes messages in $\{0,1\}^{2 k}$ and outputs signatures in $\{0,1\}^{\text {poly }(k)}$. We use $\left(G^{\prime}, \sigma^{\prime}, V^{\prime}\right)$ and the hash function $h$ to construct a new stateless signature scheme $\Sigma=(G, \sigma, V)$. We let $G=G^{\prime}$.

The signature function $\sigma:\{0,1\}^{k} \times\left(\mathbb{Z}_{p}^{*}\right)^{\alpha+1} \rightarrow\{0,1\}^{\text {poly }(k)}$ :

$$
\sigma\left(S K, r_{1} \circ \cdots \circ r_{\alpha+1}\right)=\sigma^{\prime}\left(S K, h\left(r_{1}, \ldots, r_{\alpha+1}\right)\right)
$$

The verification function $V:\{0,1\}^{k} \times\left(\mathbb{Z}_{p}^{*}\right)^{\alpha+1} \times\{0,1\}^{\text {poly }(k)} \rightarrow\{0,1\}$ :

$$
V\left(V K, s, r_{1} \circ \cdots \circ r_{\alpha+1}\right)=V^{\prime}\left(V K, s, h\left(r_{1}, \ldots, r_{\alpha+1}\right)\right)
$$

We further define $\sigma$ on input from $\left(\mathbb{Z}_{p}^{*}\right)^{\beta}$, where $\beta<\alpha+1$ as follows: $\sigma\left(r_{1}, \ldots, r_{\beta}\right)=\sigma^{\prime}\left(h\left(0, \ldots, 0, r_{1}, \ldots, r_{\beta}\right)\right) . V$ extends in the obvious way.

Lemma 2. $\Sigma=(G, \sigma, V)$ from Construction 1 is a secure stateless signature scheme under the discrete logarithm assumption:

$$
\operatorname{InSec}_{\Sigma}^{\mathrm{sig}}(t, q, k) \leq \operatorname{InSec}_{\Sigma^{\prime}}^{\mathrm{sig}}(t+O(q), q, k)+\mathbf{D L}(t+O(q), k) .
$$

Proof. The intuition behind the proof is that any adversary that can attack $\Sigma$ can be used to either attack the underlying signature scheme or calculate discrete logarithms. See full paper for details.

We use the signature scheme from Construction 1 to construct a distribution $D_{V K}$ over the alphabet $\left\{\mathbb{Z}_{p}^{*} \times\{0,1\}^{\text {poly }(k)}\right\}^{*}$, where $p$ is a $k$ bit prime and $\operatorname{poly}(k)$ is the length of a signature in $\Sigma$. Each document consists of an element in $\mathbb{Z}_{p}^{*}$ and a signature on the previous $\alpha+1$ elements.

Construction 2 (Pathological Distribution $\left.D_{V K}\right)$. Let $\Sigma=(G, \sigma, V)$ be a secure stateless signature scheme from Construction 1 . We use $G$ to generate the keys $(S K, V K)$ and index distribution $D_{V K}$ via the public verification key. If $d_{i}$ is the ith document, then $d_{i}=r_{i} \sigma\left(S K, r_{i-\alpha} \circ \cdots \circ r_{i}\right)$, where $r_{i}$ is chosen randomly from $\mathbb{Z}_{p}$. The output of $D_{V K}\langle\lambda, n\rangle$ looks like:

$$
\begin{aligned}
D_{V K}\langle\lambda, n\rangle \rightarrow & r_{1} \sigma\left(S K, r_{1}\right) \\
& \circ r_{2} \sigma\left(S K, r_{1} \circ r_{2}\right) \circ \cdots \\
& \cdots \circ r_{\alpha+1} \sigma\left(S K, r_{1} \circ r_{2} \circ \cdots \circ r_{\alpha+1}\right) \circ \cdots \\
& \cdots \circ r_{n} \sigma\left(S K, r_{n-\alpha} \circ \cdots \circ r_{n}\right)
\end{aligned}
$$

We define $\sigma_{i}=\sigma\left(S K, r_{i-\alpha}, \ldots, r_{i}\right)$.

Definition $5(\Gamma)$. Suppose we query $D_{V K}\langle\lambda, n\rangle q$ times and record the result on tape $Q$. We define the probability that any one sequence $r_{1}, \ldots, r_{d}$ appears two or more times in $Q$ as $\Gamma(d, n, q, k)$.

Lemma 3. $\Gamma(d, n, q, k)$ is a negligible function in $k$.

Proof. The proof relies on the fact that $\left|\mathbb{Z}_{p}\right|=\Theta\left(2^{k}\right)$. See full paper for details. 


\subsection{Pathology of the Distribution}

We now show that any computationally bounded stegosystem for $D_{V K}$ is guaranteed to be caught with overwhelming probability.

Theorem 4. Let $\mathcal{S}$ be an arbitrary probabilistic polynomial time stegosystem for distribution $D_{V K}$ that has a database of $q_{1}$ covertexts of length $n$ generated by $D_{V K}\langle\lambda, \cdot\rangle$ and is allowed to make $q_{2}$ queries to $D_{V K}^{\alpha-1}\langle\cdot, 1\rangle$. Suppose it takes $\mathcal{S}$ time $t$ to generate a stegotext of length $N>\alpha$. Then there exists an adversary that can distinguish $\mathcal{S}$ from $D_{V K}$ with probability $1-\nu(k)$, for a negligible function $\nu$. The adversary uses only the verification key $V K$ and $q_{1}+1$ samples from the oracle of length $N$ each; it runs in time $O\left((t+N)\left(q_{1}+1\right)\right)$.

Remark 6 . The stegosystem needs to forge signatures if it wants to generate more than $q_{1}$ distinct stegotexts. All the adversary does is ask for $q_{1}+1$ samples and checks them for duplicates and/or invalid signatures.

We will prove Theorem 4 in three steps. First we will construct an oracle $D *{ }_{V K}^{\alpha-1}$ that is information theoretically indistinguishable from $D_{V K}^{\alpha-1}\langle\cdot, 1\rangle$. Then we will show that a stegosystem whose only resource is $D *_{V K}^{\alpha-1}$ cannot create stegotexts longer than $\alpha$ with more than negligible probability. Finally, we will augment the stegosystem by giving it access to $D_{V K}\langle\lambda, \cdot\rangle$ and prove Theorem 4 by showing that it still cannot generate new stegotexts.

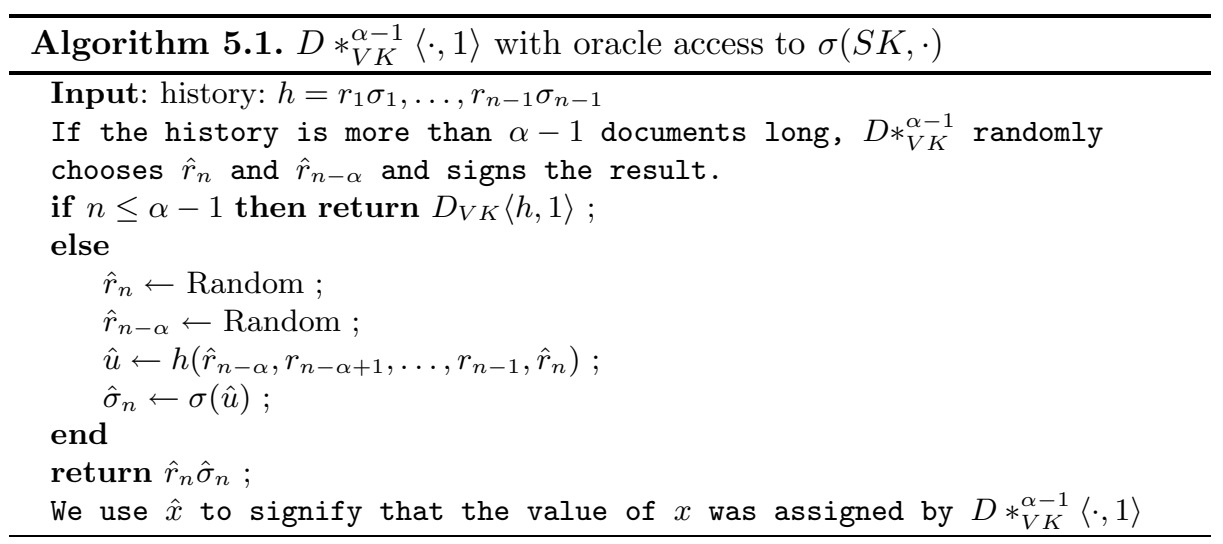

Lemma 4. Consider $D *_{V K}^{\alpha-1}\langle\cdot, 1\rangle$ (Algorithm 5.1). $D *_{V K}^{\alpha-1}\langle\cdot, 1\rangle=D_{V K}^{\alpha-1}\langle\cdot, 1\rangle$.

Proof. Lemma 4 follows from the information-theoretic hiding property of $h$, see full paper for proof.

Lemma 5. $D_{V K}$ is strictly $\alpha$-memoryless.

Proof. Lemma 5 follows from Lemma 4, see full paper for proof. 
Lemma 6. Let $\mathcal{S}$ be any stegosystem that has oracle access to $D_{V K}^{\alpha-1}\langle\cdot, 1\rangle$, but with no direct access to $D_{V K}-$ i.e. $\mathcal{S}$ does not know $S K$ and has no oracle access to $\sigma(S K, \cdot)$. Suppose it takes $\mathcal{S} t$ time and $q$ queries to $D_{V K}^{\alpha-1}\langle\cdot, 1\rangle$ to output a stegotext $s=r_{1} \sigma_{1} \circ \cdots \circ r_{n} \sigma_{n}$ of length $n>\alpha$. Then there exists an efficient adversary that can distinguish $\mathcal{S}$ from $D_{V K}$ with overwhelming probability using only one sample of length $\alpha$ and running in time $O(t)$ :

$$
\operatorname{InSec}_{\mathcal{S}, D}^{\text {cha }}(t, 1, \alpha+1, k) \geq 1-\operatorname{InSec}_{\Sigma}^{\text {sig }}(t+O(1), q, k)-\mathbf{D L}(t+O(q), k)
$$

Furthermore, $\forall i>\alpha$, the probability that an arbitrary signature $\sigma_{i}$ is valid is at most:

$$
\operatorname{InSec}_{\Sigma}^{\mathrm{sig}}(t+O(1), q, k)+\mathbf{D L}(t+O(q), k) .
$$

Proof. Assume we have a secure stegosystem $\mathcal{S}$ with no direct access to $D_{V K}$. We construct an adversary $A$ that uses $\mathcal{S}$ to forge signatures or calculate discrete $\operatorname{logs}$. $A$ tells $S$ to generate a single stegotext of any length $n>\alpha$. While $\mathcal{S}$ is working, $A$ intercepts all of $\mathcal{S}$ 's queries to $D_{V K}^{\alpha-1}\langle\cdot, 1\rangle$ and redirects them to $D *_{V K}^{\alpha-1}\langle\cdot, 1\rangle$. Finally, $\mathcal{S}$ outputs a stegotext $s=r_{1} \sigma_{1} \circ r_{2} \sigma_{2} \circ \cdots \circ r_{n} \sigma_{n}$.

Choose any $i>\alpha$. We have three cases to consider:

1. If $\sigma_{i}$ is not a valid signature on $r_{i-\alpha} \circ \cdots \circ r_{i}$ then the stegosystem is insecure.

The probability that this happens is $\operatorname{InSec}_{\mathcal{S}, D}^{\text {cha }}(t+O(1), 1, n, k)$.

2. If $\sigma_{i}$ is a valid signature on $r_{i-\alpha} \circ \cdots \circ r_{i}$ and it was not generated by $D *_{V K}^{\alpha-1}$ $\langle\cdot, 1\rangle$ then $\mathcal{S}$ violated the security of $\Sigma$. The probability that this happens is $\operatorname{InSec}_{\Sigma}^{\mathrm{sig}}(t+O(1), q, k)$.

3. If $\sigma_{i}$ is a valid signature that was generated by $D *_{V K}^{\alpha-1}\langle\cdot, 1\rangle$ then we use $\mathcal{S}$ and

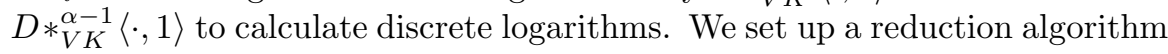

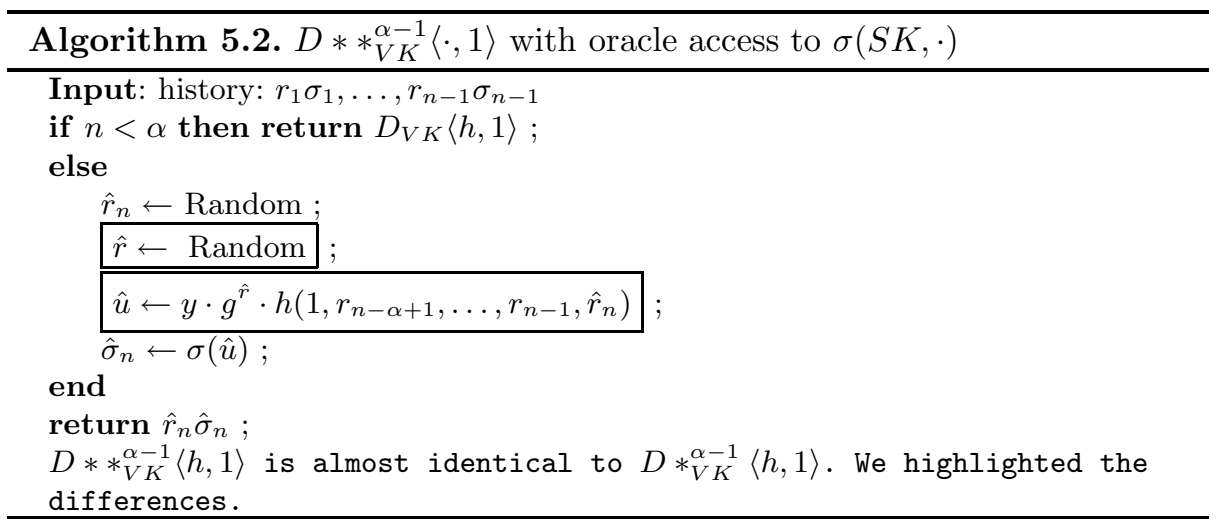

that uses the stegosystem as a black box and controls the actions of $D_{V K}^{\alpha-1}\langle\cdot, 1\rangle$. The reduction would get a challenge string $y=g^{x}$, where $g$ is a generator of the group $G$ and $x$ is unknown. Next, the reduction would ask the stegosystem to generate a stegotext. Whenever the stegosystem queries $D_{V K}^{\alpha-1}\langle\cdot, 1\rangle$, the 
reduction would redirect the call to $D * *_{V K}^{\alpha-1}\langle\cdot, 1\rangle$. Algorithm [5]2 shows how $D * *_{V K}^{\alpha-1}\langle\cdot, 1\rangle$ inserts $y$ into every signature. $D * *_{V K}^{\alpha-1}\langle\cdot, 1\rangle$ ensures that the returned signature $\hat{\sigma}_{n}$ is valid only if $r_{n-\alpha}=\log _{g}\left(y \cdot g^{\hat{r}}\right)=\log _{g}\left(g^{x+\hat{r}}\right)=$ $x+\hat{r}$, where $\hat{r}$ is chosen by $D * *_{V K}^{\alpha-1}\langle\cdot, 1\rangle$. Since the signature $\sigma_{i}$ is generated by $D * *_{V K}^{\alpha-1}\langle\cdot, 1\rangle$, we know that $s_{i-\alpha}=x+\hat{r}$. The reduction outputs $s_{i-\alpha}-\hat{r}$, thereby calculating the discrete logarithm. As a result, the probability that this case occurs is $\mathbf{D L}(t+O(q), q, k)$.

Based on our case analysis, we see that $\operatorname{InSec}_{\mathcal{S}, D}^{\text {cha }}(t, 1, n, k) \geq 1-\operatorname{InSec}_{\Sigma}^{\text {sig }}(t+$ $O(1), q, k)-\mathbf{D L}(t+O(q), k)$. Substituting $n=\alpha+1$ proves the first part of Lemma 6. Furthermore, we've shown that $\forall i \geq 1$, the probability that an arbitrary signature $\sigma_{i}$ is valid is at $\operatorname{most}_{\mathbf{I n S e c}}^{\text {sig }}(t+O(1), q, k)+\mathbf{D L}(t+O(q), k)$.

Proof (Theorem 4). Assume a stegosystem $\mathcal{S}$ has a database of $q_{1}$ covertexts generated by $D_{V K}\langle\lambda, n\rangle$ and the ability to query $D_{V K}^{\alpha-1}\langle\cdot, 1\rangle q_{2}$ times. We can create an adversary $A$ that distinguishes the output of $D_{V K}$ from $\mathcal{S}$. $A$ gets $V K$ as input and permission to query a mystery oracle that is either $D_{V K}$ or $\mathcal{S}$. $A$ will ask its oracle to generate $q_{1}+1$ covertexts of length $N$. A outputs 1 if the oracle returns any duplicate or invalid covertexts. If the oracle is $D_{V K}\langle\lambda, \cdot\rangle$, then $A$ outputs 1 with probability $\Gamma\left(N, N, q_{1}+1, k\right)$ (the probability that duplicate covertexts occur). We examine what happens when the oracle is $\mathcal{S}$.

$\mathcal{S}$ can use its covertext database to generate stegotexts. Each covertext of length $n$ can generate at most 1 valid stegotext of length $N$ (the stegosystem can take an $N$ document prefix). The stegosystem cannot take an arbitrary substring of a covertext because it would have to forge a signature on the new first integer and the $\alpha$ dummy arguments.

$\mathcal{S}$ gives $A$ a list of $q_{1}+1$ stegotexts: $s^{(1)}, \ldots, s^{\left(q_{1}+1\right)}$. Each stegotext $s^{(i)}$ can be parsed as $r_{1}^{(i)} \sigma_{1}^{(i)} \circ \cdots \circ r_{N}^{(i)} \sigma_{N}^{(i)}$. $\mathcal{S}$ can easily create $q_{1}$ distinct stegotexts from its covertext dictionary. We examine how $\mathcal{S}$ generates the $q_{1}+1$ st stegotext. There are 3 cases:

1. $\mathcal{S}$ has generated a new message signature pair that is not in the covertext database and that did not come from $D_{V K}^{\alpha-1}\langle\cdot, 1\rangle$. Then $\mathcal{S}$ has broken the security of the signature scheme $\Sigma$. $\mathcal{S}$ ran in $\left(q_{1}+1\right) t$ time and made $n q_{1}+q_{2}$ queries to $\sigma(S K, \cdot)$ (via its queries to $D_{V K}\langle\lambda, \cdot\rangle$ and $D_{V K}^{\alpha-1}\langle\cdot, 1\rangle$ ). Therefore, this case occurs with probability at most $\operatorname{InSec} \operatorname{sig}_{\Sigma}^{\text {sig }}\left(\left(q_{1}+1\right) t, n q_{1}+q_{2}, k\right)$.

2. $\mathcal{S}$ used a signature generated by $D_{V K}^{\alpha-1}\langle\cdot, 1\rangle$. By Lemma 6 , we know that $\forall i, j>\alpha, \mathcal{S}$ can use $D_{V K}^{\alpha-1}\langle\cdot, 1\rangle$ to generate a valid $\sigma_{j}^{(i)}$ with probability at most $\operatorname{InSec}_{\Sigma}^{\text {sig }}\left(t+O(1), q_{2}, k\right)+\mathbf{D L}\left(t+O\left(q_{2}\right), k\right)$. Therefore, the probability that this case occurs is the total number of such signatures $(N-\alpha)\left(q_{1}+1\right)$ times the probability that any particular one was generated by $D_{V K}^{\alpha-1}\langle\cdot, 1\rangle$. This gives a total probability of: $(N-\alpha)\left(q_{1}+1\right)\left(\mathbf{I n S e c}{ }_{\Sigma}^{\text {sig }}\left(t+O(1), q_{2}, k\right)+\mathbf{D L}\left(t+O\left(q_{2}\right), k\right)\right)$

3 . The covertext database contains two identical sequences of $\alpha$ integers, thus letting $\mathcal{S}$ cut and paste two covertexts. This occurs with probability $\Gamma\left(\alpha, n, q_{2}, k\right)$. 
Adding up the probabilities from the case analysis above, we get that

$$
\begin{aligned}
\operatorname{Adv}_{\mathcal{S}, D}^{\text {cha }} & (A, k) \geq 1-\Gamma\left(N, N, q_{1}+1, k\right)-\operatorname{InSec}_{\Sigma}^{\mathrm{sig}}\left(\left(q_{1}+1\right) t, n q_{1}+q_{2}, k\right) \\
& -(N-\alpha)\left(q_{1}+1\right)\left(\mathbf{I n S e c}_{\Sigma}^{\mathrm{sig}}\left(t+O(1), q_{2}, k\right)+\mathbf{D L}\left(t+O\left(q_{2}\right), k\right)\right) \\
& -\Gamma\left(\alpha, n, q_{2}, k\right)
\end{aligned}
$$

$A$ runs in $O\left((t+N)\left(q_{1}+1\right)\right)$ time and makes $q_{1}+1$ queries of total length $N\left(q_{1}+1\right)$.

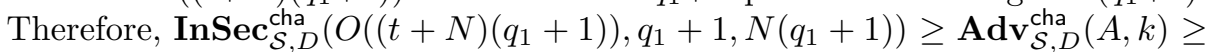
$1-\nu(k)$ for the negligible function $\nu$ defined above. This gives us the lower bound of $1-\nu(k)$ on the insecurity of $\mathcal{S}$.

\section{Conclusion}

Our results link current theoretical research to real world stegosystems. We show that a stegosystem must assume that its approximation of the covertext distribution is correct. A slight error, or a missed correlation, can lead to almost certain detection. It is impossible to leverage incomplete or incorrect information to somehow create properly distributed covertexts.

\section{Acknowledgements}

Anna Lysyanskaya is supported by NSF CAREER grant CNS-0374661. Mira Meyerovich is supported by a U.S. Department of Homeland Security (DHS) Fellowship under the DHS Scholarship and Fellowship Program and NSF grant CNS-0374661. The DHS Scholarship and Fellowship Program is administered by the Oak Ridge Institute for Science and Education (ORISE) for DHS through an interagency agreement with the U.S Department of Energy (DOE). ORISE is managed by Oak Ridge Associated Universities under DOE contract number DE-AC05-06OR23100. All opinions expressed in this paper are the authors' and do not necessarily reflect the policies and views of NSF, DHS, DOE, or ORISE.

\section{References}

[AP98] Ross J. Anderson and Fabien AP Petitcolas. On the limits of steganography. IEEE Journal on Selected Areas in Communications, 16(4):474-481, May 1998.

[BC05] Michael Backes and Christian Cachin. Public-key steganography with active attacks. In Joe Kilian, editor, Theory of Cryptography Conference Proceedings, volume 3378 of LNCS, pages 210-226. Springer Verlag, 2005.

[Cac98] Christian Cachin. An information-theoretic model for steganography. In David Aucsmith, editor, Proc. 2nd Information Hiding Workshop, volume 1525 of $L N C S$, pages 306-318. Sprinter Verlag, 1998.

[DIRR05] Nenad Dedić, Gene Itkis, Leonid Reyzin, and Scott Russell. Upper and lower bounds on black-box steganography. In Joe Kilian, editor, Theory of Cryptography Conference Proceedings, volume 3378 of LNCS, pages 227-244. Springer Verlag, 2005. 
[Gol04] Oded Goldreich. Foundations of cryptography: Volume 2, basic applications. 2004.

[HLvA02] Nicholas J. Hopper, John Langford, and Louis von Ahn. Provably secure steganography. In Moti Yung, editor, Advances in Cryptology - CRYPTO 2002, 22nd Annual International Cryptology Conference, Santa Barbara, California, USA, August 18-22, 2002, Proceedings, volume 2442 of LNCS. Springer, 2002.

[Hop04] Nicholas J. Hopper. Toward a theory of steganography. CMU Ph.D. Thesis, 2004 .

[Hop05] Nicholas J. Hopper. On steganographic chosen covertext security. In ICALP 2005, 32nd Annual International Colloquium on Automata, Languages and Programming, Lisboa, Portugal, July 11-15 2005, Proceedings, 2005.

[Le03] Tri Van Le. Efficient provably secure public key steganography. Technical report, Florida State University, 2003. Cryptography ePrint Archive, http://eprint.iacr.org/2003/156

[LK03] Tri Van Le and Kaoru Kurosawa. Efficient public key steganography secure against adaptively chosen stegotext attacks. Technical report, Florida State University, 2003. Cryptography ePrint Archive, http://eprint.iacr.org/2003/244

[LM04] Anna Lysyanskaya and Mira Meyerovich. Steganography with imperfect sampling. At: CRYPTO 2004 Rump Session, August 2004, 2004.

[LM05] Anna Lysyanskaya and Mira Meyerovich. Steganography with imperfect sampling. Technical Report ePrint Archive 2005/305, Brown University, 2005. Cryptography ePrint Archive, from http://eprint.iacr.org/ $2005 / 305$

[MLC01] Ira S. Moskowitz, Garth E. Longdon, and LiWu Chang. A new paradigm hidden in steganography. In Proceedings of the 2000 workshop on New Security Paradigms. ACM Press, 2001.

[PKSM] Kyle Petrowski, Mehdi Kharrazi, Husrev T. Sencar, and Nasir Memon. Psteg: steganographic embedding through patching. In 2005 IEEE International Conference on Acoustics, Speech, and Signal Processing.

[RR03] Leonid Reyzin and Scott Russell. Simple stateless steganography. Technical Report ePrint Archive 2003/093, Boston University, 2003. Cryptography ePrint Archive, from http://eprint.iacr.org/2003/093.

[Sal03] Phil Sallee. Model-based steganography. In IWDW, pages 154-167, 2003.

[vAH04] Louis von Ahn and Nicholas J. Hopper. Public-key steganography. In Christian Cachin and Jan Camenisch, editors, Advances in Cryptology EUROCRYPT 2004, volume 3027 of LNCS, pages 323-341. Springer Verlag, 2004. 